\title{
New Results on Nucleon Resonance Transition Form Factors
}

\author{
Volker D. Burkert, Inna Aznauryan, and the CLAS Collaboration \\ Jefferson Lab, 12000 Jefferson Avenue, Newport News, Virginia
}

\begin{abstract}
Recent measurements with CLAS at Jefferson Lab of nucleon resonance transition form factors for several lower mass states are discussed.

Keywords: Nucleon resonances, pion electroproduction, transition amplitudes, quark models

PACS: PACS: 13.60Le, 13.40Gp, 14.20Gk
\end{abstract}

\section{INTRODUCTION}

Electroexcitation of nucleon resonances has long been recognized as a sensitive tool in exploring the complex nucleon structure at varying distances scales. Mapping out the transition helicity amplitudes will tell us a great deal about the underlying quark or hadronic structure. Most of the recent data have been taken with the CLAS detector [1] using the $6 \mathrm{GeV}$ polarized electron beam at Jefferson lab. This allows to measure simultaneously the entire resonance mass region and a wide range in the photon virtuality $Q^{2}$. Several final states are measured simultaneously [2]. In this talk I discuss recent results on the extraction of transition amplitudes for several well-known states from pion electroproduction.

\section{THE $N \Delta(1232)$ TRANSITION}

An interesting aspect of nucleon structure at low energies is a possible quadrupole deformation of the lowest excited state, the $\Delta(1232)$. Such a deformation would be evident in finite values of the quadrupole transition amplitude $E_{1+}$ and $S_{1+}$, which otherwise would be equal to zero [3]. Quadrupole ratios $R_{E M}=E_{1+} / M_{1+}$ and $R_{S M}=$ $S_{1+} / M_{1+}$ are shown in Fig.1. The development of sophisticated phenomenological analysis methods $[4,5]$ over the past decade resulted in a consistent picture for these quantities. $R_{E M}$ remains negative, small and nearly constant in the entire range $0<Q^{2}<$ $6 \mathrm{GeV}^{2}$. There are no indications that leading pQCD contributions are important, which would require $R_{E M} \rightarrow+1$ [6]. The longitudinal quadrupole ratio $R_{S M}$ also remains negative, but its magnitude rises strongly with increasing $Q^{2}$. Simultaneous description of both $R_{E M}$ and $R_{S M}$ is achieved with dynamical models that include pion-nucleon interactions explicitly, supporting the idea that most of the quadrupole strength in the $N \Delta(1232)$ transition is due to meson effect $[7,8]$.

${ }^{1}$ now at Yerevan Physics Institute, Yerevan, Armenia 

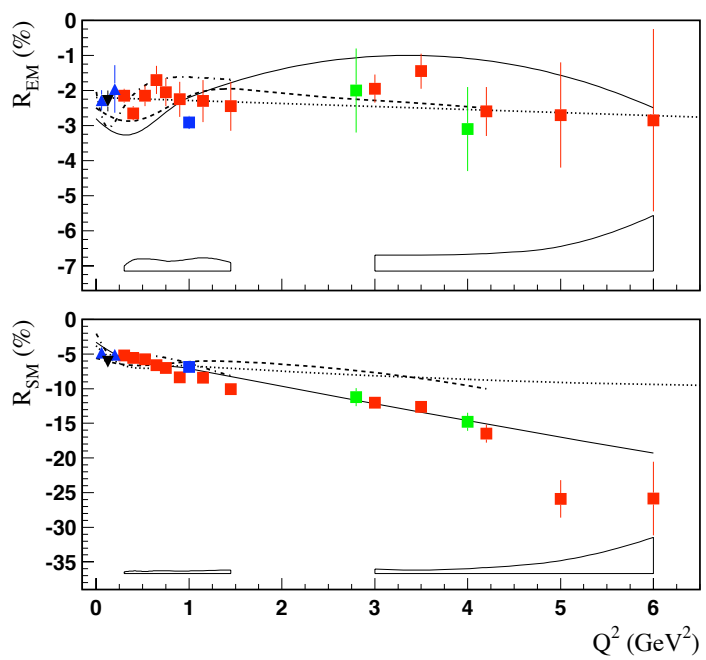

FIGURE 1. $R_{E M}$ and $R_{S M}$ from $p \pi^{\circ}$ electroproduction. $p\left(e, e^{\prime} p\right) \pi^{0}$. Data from $[9,10,12,16,18,19]$.

\section{THE ROPER RESONANCE - ONE PUZZLE RESOLVED}

The standard constituent quark model which describes this state as a radial excitation of the nucleon, has difficulties to describe basic features such as the mass, photocouplings, and $Q^{2}$ evolution. This has prompted the development of alternative models involving gluon fields [13], or meson-baryon degrees of freedom [14, 15]. Given these different theoretical concept for the structure of the state, the question "what is the nature of the Roper state?" has been a focus of the $N^{*}$ program with CLAS. The state is very wide, and pion electroproduction data covering a large range in the invariant mass $\mathrm{W}$ with full center-of-mass angular coverage are key in extracting the transition form factors. As
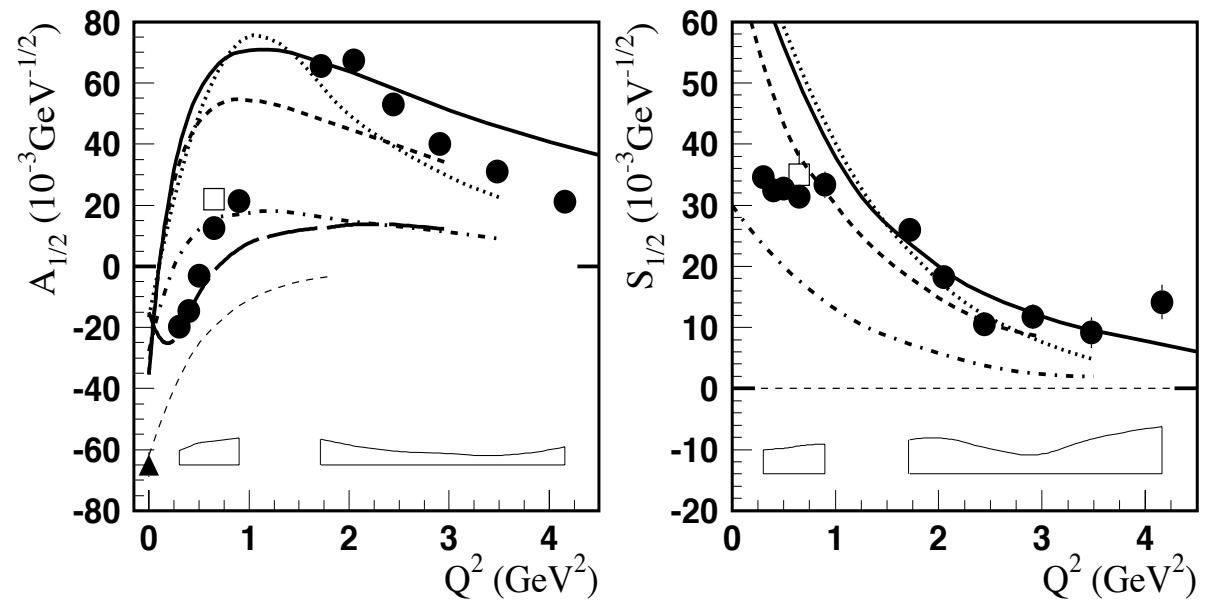

FIGURE 2. Transverse electrocoupling amplitude for the Roper $P_{11}(1440)$ (left panel). The full circles are the new CLAS results. The squares are previously published results of fits to CLAS data at low $Q^{2}$. The right panel shows the longitudinal amplitude. The bold curves are all relativistic light front quark model calculations [27]. The thin dashed line is for a gluonic excitation[13]. 
an isospin $I=\frac{1}{2}$ state, the $P_{11}(1440)$ couples more strongly to $\mathrm{n} \pi^{+}$than to $\mathrm{p} \pi^{\circ}$. Also contributions of the high energy tail of the $\Delta(1232)$ are much reduced in that channel due to the $I=\frac{3}{2}$ nature of the $\Delta(1232)$. Previous studies [11] have mostly used the $p \pi^{0}$ final state often resulting from measurements that focussed on the $\Delta(1232)$ mass region. This analysis included new high statistics $n \pi^{+}$data that covered the entire mass region up to $W=1.7 \mathrm{GeV}$.

A large sample of differential cross sections and polarization asymmetry data $[16,17$, $21,22,20,23,24]$ from CLAS have been analyzed using the fixed-t dispersion relations approach and the unitary isobar model. The transverse and longitudinal electrocoupling amplitudes $A_{1 / 2}$ and $S_{1 / 2}$ of the transition to the $N(1440) P_{11}$ resonance are extracted from fits $[25,26]$ to these data, and are shown in Fig. 2.

At the real photon point $A_{1 / 2}$ is negative, rises quickly with $Q^{2}$, and changes sign near $Q^{2}=0.5 \mathrm{GeV}^{2}$. At $Q^{2}=2 \mathrm{GeV}^{2}$ the amplitude has about the same magnitude but opposite sign as at $Q^{2}=0$. It slowly falls off at high $Q^{2}$. This remarkable behavior of a sign change with $Q^{2}$ has not been observed before for any nucleon form factor or transition amplitude. The longitudinal coupling $S_{1 / 2}$ is smaller than the transverse one. At high $Q^{2}$ both amplitudes are qualitatively described by the light front quark models, which strongly suggests that at short distances the Roper behaves indeed as expected from a radial excitation of the nucleon. The low $Q^{2}$ behavior is not well described by the LF quark models and all fall short of describing the amplitude at the photon point. This suggests that important contributions, e.g. meson-baryon interactions describing the large distances behavior, are missing.
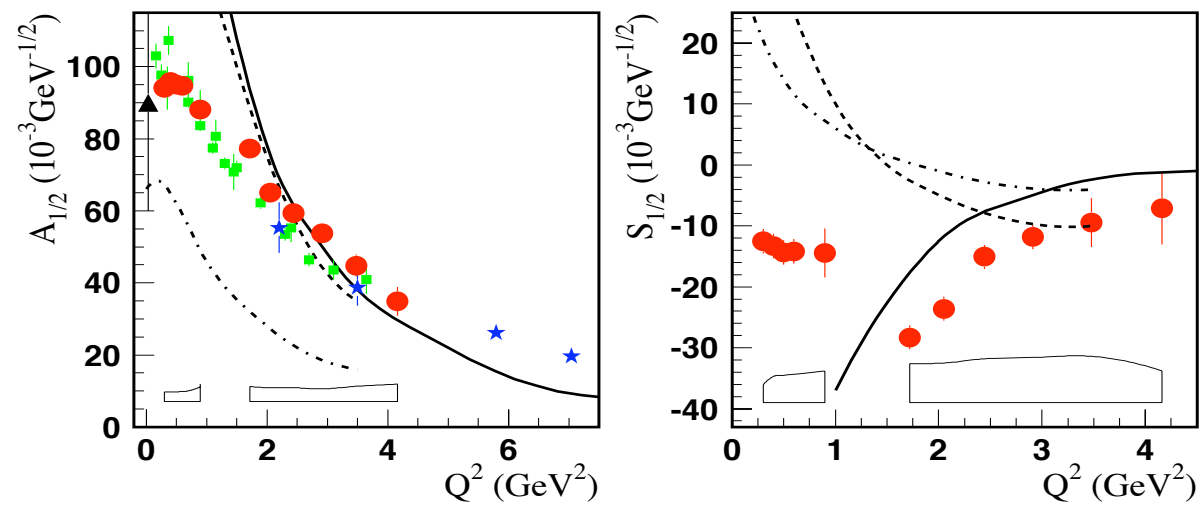

FIGURE 3. The transition amplitude $A_{1 / 2}$ (left) for the $S_{11}(1535)$. The full circles are from the analysis of the CLAS $n \pi^{+}$and $p \pi^{\circ}$ data[25, 26]. The other data are from the analysis of $p \eta$ data [28, 29, 30, 31]. The curves represent constituent quark model calculations of [33] (dashed), [34] (dashed-dotted), and [35] (solid).

\section{THE $N(1535) S_{11}$ STATE}

This state has been studied extensively in the $p \eta$ channel, where it appears as an isolated resonance near the $N \eta$ threshold. Phenomenological analyses of data from CLAS [28, 29] and Hall C $[30,31]$ have resulted in the $Q^{2}$ evolution of the transverse transition amplitude $A_{1 / 2}$ from $\eta$ electroproduction data. However, there are two re- 
maining important uncertainties that need to be examined. The first uncertainty is due to the branching ratio of the coupling $S_{11}(1535) \rightarrow p \eta$. The PDG [37] gives ranges of $\beta_{N \eta}^{P D G}=0.45-0.60$ and $\beta_{N \pi}^{P D G}=0.35-0.55$, which adds a large uncertainty to the resulting helicity amplitudes. Since this state has very small coupling to channels other than $N \eta$ and $N \pi$, a measurement of the reaction $e p \rightarrow e \pi^{+} n$ can reduce this uncertainty. Adjusting $\beta_{N \pi}=0.48$ and $\beta_{N \eta}=0.46$ brings the two data sets into excellent agreement, as shown in Fig. 3. The second uncertainty comes from the lack of precise information on the longitudinal coupling. This contribution is usually neglected when analyzing the $p \eta$ channel. An important advantage of the $N \pi$ channel is that it is also sensitive to the longitudinal transition amplitude $S_{1 / 2}$ resulting from a significant $s-p$ wave interference with the nearby $p$-wave amplitude of the $P_{11}(1440)$. Since the $P_{11}(1440)$ does not couple to $p \eta$, this channel has very little sensitivity to the $S_{1 / 2}$ amplitude.

\section{HELICITY STRUCTURE OF THE $D_{13}(1520)$}

A longstanding prediction [32] of the dynamical constituent quark model is the helicity switch from the dominance of the $A_{3 / 2}$ amplitude at the photon point to $A_{1 / 2}$ dominance at $Q^{2}>1 \mathrm{GeV}^{2}$. Indications of such behavior have been observed in previous analysis [2, $11]$, but analyses have been hampered by incomplete kinematical coverage of data, and the scarceness of $n \pi^{+}$data, which are most sensitive to the excitation of the state. The new CLAS data have largely eliminated this shortcoming. Figure 4 shows the CLAS
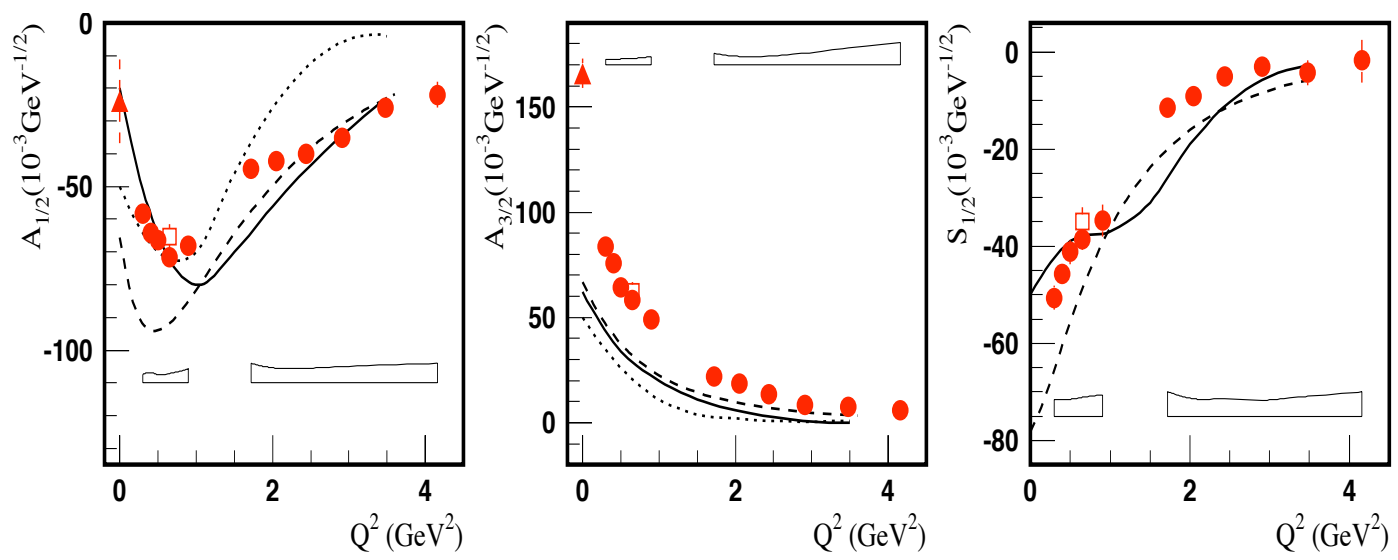

FIGURE 4. Electrocoupling amplitudes $A_{1 / 2}$ (left) and $A_{3 / 2}$ (middle) and $S_{1 / 2}$ (right) for the $D_{13}(1520)$. Model curves as in Fig.2.

results for the electrocouplings. The $A_{3 / 2}$ amplitude is large at the real photon point and decreasing rapidly in strength with increasing $Q^{2} . A_{1 / 2}$ is small at the photon point and increases rapidly in magnitude with increasing $Q^{2}$. At high $Q^{2} A_{1 / 2}$ becomes the dominant amplitude, which confirms the early prediction of the constituent quark model. 


\section{CONCLUSIONS}

The past decade of experimental research on the electroproduction of pseudoscalar mesons, has led to consistent set of data on transition amplitudes for several of the lower mass excited states of the nucleon. This set of the most precise amplitudes to date allows us to put to test models of the nucleon structure in terms of the effective degrees of freedom.

This work was performed under DOE contract DE-AC05-060R23177.

\section{REFERENCES}

1. B. Mecking et al., Nucl. Instrum. Meth. A503, 513-553, 2003.

2. V. Burkert and T.-S. H. Lee, Int. J. Phys. E13, 1035, 2004.

3. A. Buchmann and E. Henley, Phys. Rev. D65, 073017, 2002.

4. D. Drechsel, O. Hanstein, S. Kamalov, L. Tiator, Nucl. Phys. A 645, 145, 1999.

5. I. Aznauryan, Phys. Rev.C67,015209, 2003.

6. G. A. Warren, C.E. Carlson, Phys. Rev. D42, 3020, 1990.

7. T. Sato and T.S. Lee, Phys.Rev.C63, 055201, 2001.

8. S.S. Kamalov and S.N. Yang, Phys. Rev. Lett. 83, 4494, 1999.

9. S. Stave et al., arXiv:0803.2496 [hep-ex], N.F. Sparveris et al., Phys. Lett. B561, 102, 2007.

10. N.F. Sparveris et al., Phys. Rev. Lett. 94, 022003, 2005.

11. D. Drechsel, et al., Eur. Phys. J.A34, 69, 2007.

12. V.V. Frolov et al., Phys.Rev.Lett.82:45-48, 1999.

13. Z.P. Li, V. Burkert, Zh. Li; Phys .Rev. D46, 70, 1992.

14. F. Cano and P. Gonzales, Phys. Lett. B431, 270, 1998.

15. O. Krehl, et al., Phys.Rev.C62,025207, 2000.

16. K. Joo, et al, Phys. Rev. Lett. 88, 122001, 2002.

17. K. Joo et al., Phys. Rev. C68, 032201, 2003.

18. M. Ungaro et al., Phys. Rev. Lett.97, 112003, 2006.

19. J.J. Kelly et al., Phys. Rev. Lett.95, 102001, 2005.

20. H. Egiyan at al., Phys.Rev.C73,025204, 2006.

21. K. Joo et al., Phys. Rev. C70, 042201, 2004.

22. K. Joo et al., Phys. Rev. C72, 058202, 2005.

23. A. Biselli et al., Phys. Rev. C78, 045204, 2008.

24. K. Park et al., Phys.Rev.C77,015208, 2008.

25. I. Aznauryan et al., Phys. Rev. C71, 015201; Phys. Rev. C72, 045201, 2005.

26. I. Aznauryan et al. (CLAS), Phys. Rev. C78, 045209, 2008.

27. For an overview, see: I. Aznauryan, Phys. Rev. C76, 025212, 2007.

28. R. Thompson et al., Phys. Rev. Lett. 86, 1702, 2001,

29. H. Denizli, Phys. Rev. C76, 015204, 2007.

30. C.S. Armstrong et al., Phys. Rev. D60, 052004, 1999.

31. M.M. Dalton et al., arXiv:0804.3509 [hep-ex]

32. F.E. Close and F.J. Gilman, Phys. Lett. B 38, 541, 1972

33. S. Capstick and B.D. Keister, Phys. Rev. D51, 3598, 1995.

34. E. Pace, G. Salmé, and S. Simula, Few Body Syst. Suppl. 10, 407, 1999.

35. V.M. Braun, et al., arXiv:0902.3087 (hep-ph)

36. M. Warns, et al., Z. Phys. C45, 627, 1990.

37. Review of Particle Physics, C. Amsler et al., Phys. Lett. B 667, 2008. 